\title{
PC-TES: Catalogando iniciativas de Pensamento Computacional *
}

\author{
Braz Araujo da Silva Junior ${ }^{1}$, Simone André da Costa Cavalheiro ${ }^{1}$, Luciana Foss ${ }^{1} 1$ \\ ${ }^{1}$ Programa de Pós-Graduação em Computação - Universidade Federal de Pelotas \\ CEP 96.010-610 - Pelotas - RS - Brazil \\ \{badsjunior, simone.costa, lfoss\}@inf.ufpel.edu.br
}

\begin{abstract}
This paper details a system to describe skills, resources and studies under the context of computational thinking in a standardized way. It is the initial part of an effort to connect the cientific community to the informal one, making them interact and reuse the byproducts of the work of each other. To define its structures there were considered concepts from psychometrics, tools of pedagogical management, educational approaches and design of experiments.
\end{abstract}

Resumo. Este artigo detalha um sistema para descrever habilidades, recursos e estudos sob o contexto do pensamento computacional de maneira padronizada. Ele é a parte inicial de um esforço para conectar a comunidade científica à informal, fazendo-as interagir e reaproveitar os produtos gerados pelos trabalhos de ambas. Para a definição das estruturas são considerados conceitos da psicometria, ferramentas de gestão pedagógica, abordagens educacionais e de design experimental.

\section{Introdução}

Pensamento Computacional (PC) pode ser um termo confuso, principalmente para leigos e iniciantes na área. O termo é associado por parte dos educadores à introdução de tecnologias em sala de aula, como programação e robótica [Haines et al. 2019, Mota and Neves 2020]; também aparece como sinônimo de ciência da computação básica/para iniciantes [Lu and Fletcher 2009]; e comumente é tratado como um conjunto de habilidades de propósito geral que auxiliam no processo de resolução de problemas [Alencar et al. 2020, Lopes et al. 2020, Denning and Tedre 2019]. Muitos trabalhos propõem frameworks de PC com seu próprio conjunto de habilidades, além disto, o que significa e o que está incluso em cada uma destas habilidades acaba variando de autor para autor, como evidenciado em uma revisão sistemática que expõe os termos que são relacionados ao PC em 28 trabalhos [Silva Junior 2020]. Eis que convergência surge como uma carência da área. Embora existam conceitos predominantes na literatura sobre o que está incluso no PC (como abstração, algoritmos, decomposição e reconhecimento de padrões), ainda há um amplo debate sobre o que realmente é PC [Román-González et al. 2019]. Como pontuado por [Kalelioglu et al. 2016], apesar das definições terem evoluído após o artigo que reacendeu a discussão e deu luz ao termo [Wing 2006], ainda não há uma definição consensual.

* O presente trabalho foi realizado com apoio da Coordenação de Aperfeiçoamento de Pessoal de Nível Superior - Brasil (CAPES) - Código de Financiamento 001. Projeto realizado com o apoio da PREC e PRPPG / UFPel. 
Percebe-se portanto que é um grande desafio para os trabalhos que se propõem a conceituar, definir ou explicar PC concordarem plenamente à partir da listagem de eixos, pilares, habilidades fundamentais ou ainda dimensões do PC. Assim, este trabalho apresenta uma estratégia diferente para que pesquisadores, professores, líderes e entusiastas do PC possam trabalhar de maneira compatível e coerente, atingindo a convergência que se falha em alcançar através de definições gerais. Tomando a direção contrária dos trabalhos citados acima, ao invés de partir de conceitos-chave, abrangentes por natureza, este trabalho preocupa-se em listar as especificidades, investigar de maneira pontual o que vêm sendo trabalhado dentro deste âmbito do PC. Este esforço também se dá no sentido de trazer regularidade e padronização às atividades, bem como facilitar a centralização de recursos e conhecimentos, para que a pesquisa e o ensino-aprendizagem de PC possam ser melhor organizados, difundidos e postos em prática. Neste sentido, o trabalho aqui reportado propõe e discute um modelo para descrever de maneira padronizada (catalogar) o que é trabalhado no contexto do PC. Marca-se com ele o início de um projeto colaborativo com o intuito de unir esforços de pesquisadores e educadores, centralizando recursos e possibilitando a troca de informações e experiências acerca do PC.

O restante deste artigo está organizado como segue. A subseção 1.1 situa este trabalho dentro de um projeto colaborativo de pesquisa científica com participação pública, discute esse modelo de pesquisa e trabalhos relacionados ao projeto. A subseção 1.2 declara trabalhos que serviram como base para a concepção da estrutura de dados proposta. A seção 2 descreve a estrutura do sistema de catalogação. A seção 3 mostra uma catalogação de exemplo para ilustrar sua utilização, classificando parte do que é trabalhado em um artigo publicado como os componentes descritos na seção 2. A seção 4 conclui o artigo discutindo as contribuições do sistema para a área e os próximos passos que podem ser dados para que estas contribuições sejam potencializadas e consolidadas.

\subsection{Um pacto pelo Pensamento Computacional}

Este trabalho inaugura o projeto "Partnership for Advancing Computational Thinking (PACT)", onde é almejada a construção de uma ponte entre o ambiente acadêmico e o informal. Por um lado, resgatando e divulgando para além da comunidade científica recursos de estudos relatados em artigos e periódicos. E pelo outro lado, guiando e incentivando o relato de iniciativas informais ou mesmo comerciais que não seriam publicadas nos meios científicos para que pesquisadores tenham acesso a estes dados. Popularmente conhecido como "Citizen science", o modelo de pesquisa com participação pública conta com cidadãos ao redor do mundo para adquirirem e gerarem dados para pesquisas através de alguma rede de colaboração [Bonney et al. 2009]. Esforços neste formato já tem mostrado resultados promissores, são frequentes e bem estabelecidos em áreas como astronomia e biologia, mas ainda são escassos em outras áreas [MacDonald et al. 2015].

Não é de conhecimento dos autores deste artigo nenhum trabalho identificado como projeto de "Citizen science" na área da educação. Mas é defendido que: assim como qualquer cidadão pode contribuir com pesquisas de controle e monitoramento ambiental tirando fotos de espécies de animais encontradas em determinada área, também pode haver contribuição significativa para a área da educação vinda de educadores nãopesquisadores que realizam atividades e estão em contato diário com alunos (ainda que estas atividades não estejam contempladas em um projeto de pesquisa, nem contém um planejamento científico pleno para sua realização). Para isto, basta que os dados de tais 
atividades, relatados informalmente, sejam normalizados por um sistema para adequá-los a análises técnico-científicas. Adicionalmente os educadores também podem se beneficiar desta interação, tendo acesso a recursos desenvolvidos em ambiente acadêmico.

Portanto, o projeto PACT é um esforço que traz o conceito "Citizen science" para a educação em computação, para reunir, padronizar, compartilhar, dar suporte e orientar pesquisas, planos, projetos e iniciativas acerca do PC promovendo e mediando a troca de informações entre a comunidade científica e educadores não-pesquisadores. O objetivo final do PACT é iniciar e estabelecer uma rede colaborativa de referência e compartilhamento de saberes, experiências, materiais e ferramentas para fomentar o ensinoaprendizagem de PC. O projeto conta com os seguintes eixos e seus respectivos fins: estrutural, para mediar a comunicação, concentrar e disponibilizar conteúdo proveniente de ambos os públicos; científico, para fomentar a catalogação a partir das fontes de origem científica, o que será realizado por meio de revisões sistemáticas e meta-análises da literatura em PC; e informal, para fomentar a catalogação a partir das fontes de origem não-científica, o que será realizado por meio de uma plataforma para educadores inserirem dados sobre suas intervenções. Neste artigo é tratada a questão da estrutura do sistema "Pensamento Computacional em Tópicos, Expressões e Sensibilidades (PC-TES)", que visa descrever de forma padronizada informações relevantes sobre PC para que elas possam: servir de Modelo Entidade Relacionamento (MER) para o banco de dados do eixo estrutural; guiar as revisões e análises do eixo científico; e definir os campos que a plataforma para educadores irá disponibilizar no eixo informal.

\subsection{Concepção da estrutura}

Para a definição dessa estrutura, tomou-se como base de inspiração dois esforços relacionados à descrição de habilidades, especialmente no contexto da educação: a Base Nacional Comum Curricular (BNCC) [Brasil 2017] e o Currículo de Tecnologia e Computação (CTC) [Raabe et al. 2018]. No Brasil, a BNCC cumpre o papel de orientar a formulação dos projetos Político-Pedagógicos das escolas, trazendo em sua estrutura listas de competências e habilidades esperadas como resultado do ensino-aprendizagem ao longo da educação básica. Particularmente, há diversas referências explícitas ao PC e a conceitos relacionados, como "cultura digital", "Tecnologias Digitais da Informação e Comunicação (TDIC)", "redes sociais" e "algoritmo" ao longo da BNCC, principalmente relacionadas à área da matemática [Vicari et al. 2018]. Mas nem o PC nem a computação (ou ciência da computação) são considerados áreas ou componentes curriculares, com suas próprias competências específicas e habilidades, como sugerido pela Sociedade Brasileira da Computação (SBC) [SBC 2018] e Centro de Inovação para a Educação Brasileira (CIEB) [CIEB 2018]. Já o CTC cumpre papel semelhante, mas direcionado a três eixos: cultura digital, tecnologia digital e pensamento computacional. Além de organizar habilidades, o CTC disponibiliza informações para dar suporte e orientação aos professores e gestores interessados em implementações. São informações sobre como pode ser feita a avaliação, materiais de referência e os chamados níveis de maturidade da escola e do docente, que indicam respectivamente os requisitos de infraestrutura e preparo. A partir do estudo da estrutura da BNCC e do CTC, o PC-TES foi concebido incorporando algumas características destes instrumentos de gestão pedagógica. 


\section{Composição do sistema PC-TES}

A função do sistema PC-TES para o PACT é normalizar informações de diferentes naturezas (científica e informal) e garantir que os dados armazenados atendam ao interesse de ambas as fontes. Considera-se que o interesse de cientistas que usarão o sistema gira em torno de relatos de intervenções/aplicações informais de iniciativas do PC. Aqui essas intervenções são modeladas como estudos, a fim de facilitar a utilização dos dados para fins de pesquisa. Do outro lado, considera-se que o interesse dos educadores nãopesquisadores gira em torno de achar o que e como trabalhar dentro do contexto do PC. O PC-TES modela recursos (como materiais e ferramentas que podem ser utilizadas em sala de aula), associando-os às especificidades das habilidades do PC: seus "tópicos, expressões e/ou sensibilidades", para os quais usaremos a notação t/e/s.

- Tópicos são matérias, assuntos, conceitos que podem ser vistos e estudados em sala de aula. Como os componentes mais objetivos das habilidades, sua avaliação deve ser centrada em dados, textos ou quaisquer produtos gerados como respostas pelos alunos. Podem ser comparados aos objetos de conhecimento da BNCC, como "Segunda Guerra Mundial" e a parte de habilidades do CTC, como "[PC07AB01] Conhecer o conceito de grafo (...)".

- Expressões são ações planejadas, projetadas e/ou conscientes que os alunos tomam. Como os componentes que mais equilibram objetividade e subjetividade, sua avaliação deve ser centrada em ações, metodologias ou estratégias tomadas pelos alunos. Podem ser comparados a alguns dos processos cognitivos descritos pelos verbos das habilidades da BNCC, como "(EF35LP25) Criar narrativas ficcionais, com certa autonomia, utilizando detalhes descritivos (...)" e do CTC, como "[PC09AL01] Desenvolver algoritmos que utilizem recursão, compreendendo os efeitos do escopo de uma variável".

- Sensibilidades são perspectivas, percepções e/ou reflexos que os alunos desenvolvem. Como os componentes mais subjetivos das habilidades, sua avaliação deve ser centrada em reações, tempo de reação e em justificativas e análises dadas pelos alunos para suas próprias ações. Podem ser comparados a alguns (outros) processos cognitivos das habilidades da BNCC, como "(EF03MA10) Identificar regularidades em sequências ordenadas de números naturais (...)" e do CTC, como "[PC07RP01] Identificar elementos que se repetem em diferentes softwares (...)".

A escolha dos termos foi feita com o objetivo de deixar o sistema o mais intuitivo possível, para que seja natural a interpretação que: os tópicos de uma habilidade são os temas, os pontos que podem ser trabalhados para desenvolvê-la; as expressões de uma habilidade são as formas que os alunos expressam aquela habilidade através de suas ações; e as sensibilidades de uma habilidade são as coisas às quais o aluno passa a ser sensível, passa a perceber conforme desenvolve a habilidade. Seguindo uma noção alinhada com trabalhos da psicometria [Carroll et al. 1993], o sistema PC-TES considera habilidade um atributo relacionado a uma classe de tarefas, onde a intensidade desse atributo se reporta à performance geral do indivíduo nestas tarefas. Enquanto que competência é a aptidão/capacidade do indivíduo em realizar determinada tarefa. Portanto, a classe de tarefas a qual uma habilidade está relacionada no sistema PC-TES é composta pela união de todas as tarefas mencionadas nas competências de seus t/e/s. 
Competências descrevem manifestações de habilidades, ou seja, exemplos do que o aluno deve ser capaz de fazer ao desenvolver uma ou mais habilidades até determinado ponto. Como tarefas podem ter diferentes níveis de dificuldade, as competências para realizá-las também são classificadas em níveis de aptidão correspondentes. Para organizar estes níveis o PC-TES utiliza o conceito dos seis pontos críticos [Silva Junior 2020], que se baseia na sistematização do progresso em performances de [Dreyfus and Dreyfus 1980]. Esse conceito considera seis marcos no progresso da habilidade de um indivíduo em relação a uma tarefa base: o letramento, quando passa a ser capaz de realizá-la com instruções detalhadas e/ou ajuda contínua; a familiarização, quando precisa somente de instruções básicas e/ou ajuda inicial; a competência, quando não precisa mais de instruções ou ajuda; a fluência, quando passa a fazê-la em segundo plano, sem dedicar atenção exclusiva; a proficiência, quando passa a fazê-la com excelência, das melhores maneiras conhecidas; e a maestria, quando quebra recordes, apresenta desempenho acima dos conhecidos. Portanto, o nível de aptidão de uma competência é definido pelo mais alto marco (em relação à tarefa base) que o aluno já deve ter passado quando a possui. Isso é uma estimativa, deve ser usada somente como referência.
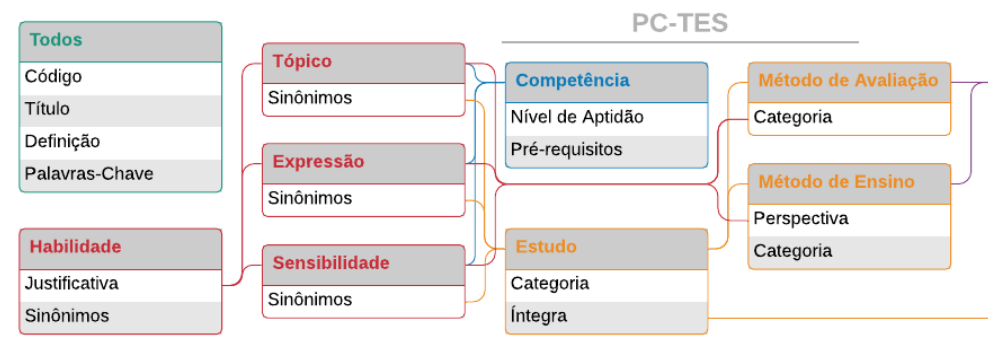

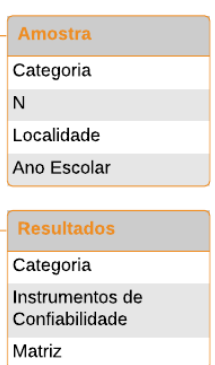

Figura 1. Visão geral do sistema PC-TES.

A Figura 1 ilustra uma visão geral dos elementos do sistema PC-TES, seus atributos e relacionamentos. Os três elementos principais do sistema são: as habilidades, para guiar o que trabalhar; os recursos, para fornecer como trabalhar; e os estudos, para demonstrar por que trabalhar. As características específicas de cada elemento são discutidas nas subseções seguintes, mas existem os atributos básicos, comuns a todos: códigos, título, definição e palavras-chave.

Códigos cumprem a função de contextualizar o elemento, passar o maior número de informações em uma curta representação. Esta é uma das características incorporadas do CTC e BNCC. Por exemplo, na BNCC é possível saber que o código EF09LI16 se refere à décima sexta (16) habilidade da Língua Inglesa para o nono (09) ano do Ensino Fundamental. E no CTC, o código PCEIAL01 se refere à primeira (01) habilidade de ALgoritmos para o Ensino Infantil no eixo do Pensamento Computacional. Já no PC-TES, o código 03T02TD09 se refere à nona (09) ferramenta (T, de Tool) Digital do segundo (02) tópico da habilidade número $\mathbf{0 3}$. A composição do código de cada elemento é detalhada na Tabela 1. Onde as linhas descrevem as sequências de caracteres que compõem o elemento identificado na primeira coluna. Estas linhas são divididas entre as opções de caractere que determinado espaço apresenta, indicando o significado de cada caractere em sua última coluna. Por exemplo, o código de um estudo é composto por um 
código t/e/s, seguido de uma letra $\mathrm{S}$ e uma letra T, R, N, Q ou P, e então uma numeração sequencial. Os termos $n 1, n 2, n 3$ se referem a diferentes numerações sequenciais, c1 e c2 a classificações e nat a natureza do t/e/s.

Tabela 1. Significado dos códigos no sistema PC-TES

\begin{tabular}{|c|c|c|c|c|c|c|c|}
\hline & n1 & nat & n2 & c1 & c2 & n3 & Significado \\
\hline $\begin{array}{l}\infty \\
\vdots \\
\infty\end{array}$ & {$[0-9]^{*}$} & $\begin{array}{l}\mathrm{T} \\
\mathrm{E} \\
\mathrm{S} \\
\end{array}$ & {$[0-9]^{*}$} & & & & $\begin{array}{l}\text { Tópico } \\
\text { Expressão } \\
\text { Sensibilidade }\end{array}$ \\
\hline 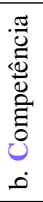 & & ligo & & C & $\begin{array}{l}\mathbf{L} \\
\mathbf{I} \\
\mathrm{C} \\
\mathrm{F} \\
\mathbf{P} \\
\mathbf{M}\end{array}$ & {$[0-9]^{*}$} & $\begin{array}{l}\text { Letramento } \\
\text { Familiaridade (no inglês, Intimacy) } \\
\text { Competência } \\
\text { Fluência } \\
\text { Proficiência } \\
\text { Maestria }\end{array}$ \\
\hline 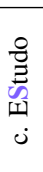 & & ligo & & $\mathbf{S}$ & $\begin{array}{l}\text { T } \\
R \\
N \\
Q \\
P \\
P\end{array}$ & {$[0-9]^{*}$} & $\begin{array}{l}\text { Teórico } \\
\text { Revisão } \\
\text { Empírico Não-experimental } \\
\text { Empírico Quasi-experimental } \\
\text { Empírico eXperimental }\end{array}$ \\
\hline 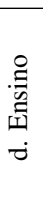 & & digo & & $\begin{array}{c}\mathrm{U} \\
\mathrm{M}\end{array}$ & $\begin{array}{l}\text { L } \\
\text { U } \\
\text { D } \\
\text { T }\end{array}$ & {$[0-9]^{*}$} & $\begin{array}{l}\text { Aluno como Usuário } \\
\text { Aluno como criador (no inglês, Maker) } \\
\text { Aula expositiva (no inglês, Lecture) } \\
\text { Atividade desplugada (no inglês, Unplugged) } \\
\text { Atividades Digitais } \\
\text { Robótica e sistemas eletrônicos (no inglês, Tinkering) }\end{array}$ \\
\hline 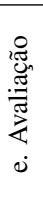 & & digo & & $\mathbf{E}$ & $\begin{array}{l}\text { O } \\
\mathrm{R} \\
\mathrm{I} \\
\mathrm{Q} \\
\mathrm{E} \\
\mathrm{S}\end{array}$ & {$[0-9]^{*}$} & $\begin{array}{l}\text { Observação } \\
\text { Rubricas } \\
\text { Entrevista (no inglês, Interview) } \\
\text { Questionário (resposta sugerida/múltipla-escolha) } \\
\text { Exame (resposta livre/dissertativa) } \\
\text { Pontuação em um sistema de regras (no inglês, Score) }\end{array}$ \\
\hline 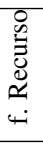 & & digo & & $\begin{array}{l}\text { A } \\
\text { T } \\
\text { P } \\
\text { G }\end{array}$ & $\begin{array}{c}\text { c2 } \\
\text { de d. } \\
\text { ou e. }\end{array}$ & {$[0-9]^{*}$} & $\begin{array}{l}\text { Material não-interativo (no inglês, } \text { Asset) } \\
\text { Ferramenta interativa (no inglês, } \text { Tool) } \\
\text { Conteúdo Prévio ou Posterior à atividade. } \\
\text { Guias, tutoriais e/ou manuais. }\end{array}$ \\
\hline
\end{tabular}

Sobre as classificações dos métodos de ensino, apontamos que metodologias ativas, movimento Maker e outros que colocam aluno como criador podem soar como novidade ou proposta, mas seguem exemplos com os quais já estamos bem familiarizados: as tradicionais apresentações de trabalhos/pesquisas (aula expositiva); montagem de robôs (atividades desplugadas); e progamação de jogos e aplicativos (atividades digitais).

Títulos cumprem a função de identificação em linguagem natural, uma palavra ou frase curta que nomeia o elemento. Apesar de parecerem triviais, especificamente no contexto do PC-TES, definir títulos adequados pode não ser uma tarefa muito fácil, uma vez que isso inclui nomear alguns conceitos que são referidos por múltiplas expressões diferentes. Portanto, além dos títulos, as habilidades, os t/e/s, contam com um campo de sinônimos, que lista as diferentes expressões que podem ser encontradas se referindo ao mesmo conceito. É importante destacar que para o sistema, o título é tratado como o termo correto para identificar o conceito. Os sinônimos são tratados sempre como alternativas, segundas opções, caso a primeira falhe em sistemas de buscas por exemplo.

Definições cumprem a função de descrever de maneira clara e objetiva o que é o elemento. Somente uma definição por elemento é admitida. O PC-TES tem como alvo especificidades, conceitos mais abrangentes devem ser usados como palavras-chave. Essas cumprem a função de indicar conceitos relacionados ao elemento, principalmente para fins de busca. Este é um componente incorporado do CTC, que as utiliza em seu 
sistema de busca em sua plataforma digital. Termos genéricos e possíveis classificações sobre os elementos devem ser utilizados como palavras-chave.

Tabela 2. Estrutura do sistema PC-TES

\begin{tabular}{|c|c|c|c|c|}
\hline Elemento & Descreve & Componente & $\mathbf{N}$ & Define \\
\hline Habilidade & $\begin{array}{l}\text { Um atributo relacionado à } \\
\text { performance geral de um indivíduo } \\
\text { em relação a uma classe de tarefas. }\end{array}$ & $\begin{array}{l}\text { Justificativa } \\
\text { Sinônimos }\end{array}$ & $\begin{array}{c}1 \\
0+\end{array}$ & $\begin{array}{l}\text { A relação com a computação. Responde "Por } \\
\text { que isto é considerado parte do PC?" } \\
\text { Outros termos que nomeiam a habilidade. }\end{array}$ \\
\hline $\begin{array}{l}\text { Expressão, } \\
\text { Tópico e/ou } \\
\text { Sensibilidade }\end{array}$ & $\begin{array}{l}\text { Os componentes teóricos (tópico), } \\
\text { ativos (expressão) e passivos } \\
\text { (sensibilidade) de habilidades. }\end{array}$ & $\begin{array}{l}\text { Habilidade } \\
\text { Sinônimos }\end{array}$ & $\begin{array}{l}1+ \\
0+\end{array}$ & $\begin{array}{l}\text { A qual(is) habilidade(s) pertence o t/e/s. } \\
\text { Outros termos que nomeiam o t/e/s. }\end{array}$ \\
\hline Competência & $\begin{array}{l}\text { Uma tarefa a qual se espera que } \\
\text { um aprendiz seja capaz de realizar } \\
\text { em determinado ponto de uma } \\
\text { habilidade. }\end{array}$ & $\begin{array}{l}\text { t/e/s } \\
\text { Pré- } \\
\text { Requisitos } \\
\text { Nível de Ap- } \\
\text { tidão }\end{array}$ & $\begin{array}{c}1+ \\
0+ \\
1\end{array}$ & $\begin{array}{l}\text { A qual(is) t/e/s pertence a competência. } \\
\text { Quais outras competências (não necessaria- } \\
\text { mente do PC) são necessárias de antemão. } \\
\text { Uma estimativa de dificuldade da tarefa con- } \\
\text { forme o sistema dos seis pontos críticos. }\end{array}$ \\
\hline Estudo & $\begin{array}{l}\text { Uma proposta ou relato (científico } \\
\text { ou informal) envolvendo t/e/s do } \\
\text { PC. O campo de definição é usado } \\
\text { para o resumo do trabalho. Os } \\
\text { estudos empíricos devem } \\
\text { relacionar ao menos uma amostra e } \\
\text { ao menos um resultado. }\end{array}$ & $\begin{array}{l}\text { Objeto } \\
\text { Categoria } \\
\text { Íntegra } \\
\text { Produtos } \\
\text { Aplicações } \\
\text { Amostras } \\
\text { Resultados }\end{array}$ & $\begin{array}{c}1+ \\
1 \\
1 \\
0+ \\
0+ \\
0+ \\
0+\end{array}$ & $\begin{array}{l}\text { Qual(is) t/e/s o estudo envolve. } \\
\text { A categoria do estudo. } \\
\text { Referência ou pdf do trabalho completo. } \\
\text { Recursos/métodos desenvolvidos/propostos. } \\
\text { Recursos/métodos utilizados. } \\
\text { Grupos de participantes do estudo. } \\
\text { Os dados gerados pelo estudo. }\end{array}$ \\
\hline $\begin{array}{l}\text { Método de } \\
\text { Ensino }\end{array}$ & $\begin{array}{l}\text { Uma forma de estimular uma } \\
\text { habilidade. }\end{array}$ & $\begin{array}{l}\text { Alvo } \\
\text { Perspectiva } \\
\text { Categoria }\end{array}$ & $\begin{array}{c}1+ \\
1 \\
1\end{array}$ & $\begin{array}{l}\text { Qual(is) t/e/s são estimulados pelo método. } \\
\text { Se o aluno é criador ou usuário. } \\
\text { A categoria do método de ensino. }\end{array}$ \\
\hline $\begin{array}{l}\text { Método de } \\
\text { Avaliação }\end{array}$ & $\begin{array}{l}\text { Uma forma de atestar (ou } \\
\text { quantificar) uma habilidade. }\end{array}$ & $\begin{array}{l}\text { Alvo } \\
\text { Base }\end{array}$ & $\begin{array}{c}1+ \\
1\end{array}$ & $\begin{array}{l}\text { Qual(is) t/e/s são avaliados pelo método. } \\
\text { No que se fundamenta a avaliação. }\end{array}$ \\
\hline Recurso & $\begin{array}{l}\text { Meios de se estimular ou avaliar } \\
\text { habilidades. }\end{array}$ & $\begin{array}{l}\text { Alvo } \\
\text { Categoria } \\
\text { Arquivos }\end{array}$ & $\begin{array}{c}1+ \\
1 \\
1+\end{array}$ & $\begin{array}{l}\text { A qual(is) método(s) o recurso dá suporte. } \\
\text { A categoria do recurso. } \\
\text { Os Arquivos de formatos diversos ou links que } \\
\text { compõem o recurso. }\end{array}$ \\
\hline Amostra & $\begin{array}{l}\text { As características de um grupo de } \\
\text { participantes de um estudo } \\
\text { empírico. }\end{array}$ & $\begin{array}{l}\text { Categoria } \\
\mathrm{N} \\
\text { Adicionais }\end{array}$ & $\begin{array}{c}1 \\
1 \\
0+\end{array}$ & $\begin{array}{l}\text { Se é grupo controle ou sujeito. } \\
\text { O número de participantes. } \\
\text { Dados adicionais como sócio-econômicos, } \\
\text { idade e localidade dos participantes. }\end{array}$ \\
\hline Resultado & $\begin{array}{l}\text { O produto de um estudo empírico. } \\
\text { O campo de definição é usado para } \\
\text { a conclusão textual dos autores. }\end{array}$ & \begin{tabular}{l|} 
Matriz \\
Tipo \\
Confiabilidade
\end{tabular} & $\begin{array}{c}1 \\
1 \\
0+\end{array}$ & $\begin{array}{l}\text { Os dados resultantes do estudo. } \\
\text { O tipo dos dados da matriz. } \\
\text { Análises e/ou métodos estatísticos usados. }\end{array}$ \\
\hline
\end{tabular}

A Tabela 2 apresenta todos os elementos do sistema PC-TES (coluna 1), suas descrições (coluna 2), os componentes de cada elemento (coluna 3), a quantidade que cada elemento pode ter de cada componente (coluna 4), e o que cada componente define (coluna 5). Componentes em roxo são referências a outros componentes. Componentes em vermelho são classificações cujo significado é apresentado com o código correspondente na Tabela 1. Para complementar as informações da tabela sobre os resultados, seguem exemplos de tipos de dados da matriz: escala contínua normalizada de $0-1$; escala discreta, como notas ABCDF; escala de Lickert; e valores Booleanos, verdadeiro ou falso. A matriz de resultados são os dados resultantes da avaliação, onde cada linha corresponde a uma intervenção em um grupo e cada coluna corresponde a um participante. Por exemplo, a matriz a seguir representa os dados de um estudo com 2 grupos de 10 participantes, um controle (C) e um sujeito (S), onde foi realizado um pré (-) e um pós (+) teste com provas valendo de 0 a 10 . A ordem das linhas obedece primeiro a ordem cronológica das intervenções, depois a prioridade é de grupos controle, e então a ordem da numeração das amostras do estudo, portanto no exemplo abaixo a ordem é C-,S-,C+,S+. 


$\begin{array}{cccccccccc}5.5 & 7.0 & 8.5 & 6.5 & 6.0 & 9.5 & 8.5 & 4.5 & 4.5 & 7.0 \\ 7.0 & 6.0 & 7.5 & 7.5 & 8.5 & 8.0 & 7.5 & 5.0 & 6.5 & 4.0 \\ 5.0 & 7.5 & 8.5 & 8.5 & 5.5 & 9.0 & 9.0 & 4.0 & 4.5 & 5.5 \\ 8.5 & 8.5 & 9.5 & 9.0 & 10.0 & 10.0 & 9.5 & 9.0 & 8.5 & 6.5\end{array}$

\section{Catalogação exemplo}

Este artigo apresentada a estrutura do sistema PC-TES, não o conteúdo que obedecerá a este formato. Porém, para fins de demonstração da utilização do sistema, é apresentada uma catalogação exemplo. Ressalta-se que o conteúdo a seguir tem fins meramente ilustrativos e não faz parte do sistema real, que será fruto de trabalhos futuros nos eixos científico e informal do PACT. Em uma utilização real do sistema, as habilidades, t/e/s, títulos e definições devem ser revistas para que sejam compatíveis e coerentes com o restante do sistema, principalmente em nível de especifidade e abrangência.

Tabela 3. Exemplo de catalogação utilizando o sistema PC-TES

\begin{tabular}{|c|c|c|c|c|}
\hline Elemento & Código & Título & Definição & Citação \\
\hline Habilidade & 01 & Decomposição & \multicolumn{2}{|c|}{$\begin{array}{l}\text { A decomposição é uma abordagem de resolução de problemas que prescreve a } \\
\text { divisão de um problema complexo em partes mais simples e mais fáceis de lidar } \\
\text { (no estilo dividir e conquistar). }\end{array}$} \\
\hline Habilidade & 02 & Generalização & \multicolumn{2}{|c|}{$\begin{array}{l}\text { A generalização (...) consiste no reconhecimento de padrões e na aplicação de } \\
\text { uma mesma solução à problemas similares. }\end{array}$} \\
\hline Expressão & $01 \mathrm{E} 01$ & Divisão & Dividir problemas em partes menores. & $\begin{array}{l}\text { (...) decompor o problema maior }(\ldots) \\
\text { em }(\ldots) \text { problemas menores }(\ldots)\end{array}$ \\
\hline Sensibilidade & 01S01 & $\begin{array}{l}\text { Processos } \\
\text { Compostos }\end{array}$ & $\begin{array}{l}\text { Reconhecer que um processo é com- } \\
\text { posto por processos menores. }\end{array}$ & $\begin{array}{l}\text { Nível } 8(\ldots) \text { : libertar o prisioneiro, der- } \\
\text { rotar o inimigo e pegar o tesouro. }\end{array}$ \\
\hline Competência & 01S01CI01 & $\begin{array}{l}\text { Fragmentação } \\
\text { para inserção }\end{array}$ & $\begin{array}{l}\text { Ao se deparar com um processo } A \text {, } \\
\text { que não pode ser feito antes nem de- } \\
\text { pois de um processo } B \text {, identificar } \\
\text { como solução: decompor } B \text { em } b_{1} \text {, } \\
b_{2}, \ldots, b_{n} \text { e inserir } A \text { entre os } b \text { s. }\end{array}$ & $\begin{array}{l}\text { o jogador não pode ir diretamente em } \\
\text { busca do tesouro, pois acabaria derro- } \\
\text { tado. Assim, precisa decompor o pro- } \\
\text { blema (...) }\end{array}$ \\
\hline Competência & 01E01CL01 & $\begin{array}{l}\text { Divisão Orien- } \\
\text { tada }\end{array}$ & $\begin{array}{l}\text { Orientar a divisão de um problema por } \\
\text { objetivos secundários que são dados } \\
\text { ao aprendiz. }\end{array}$ & $\begin{array}{l}\text { (...) decompor o problema "pegar o } \\
\text { tesouro" em dois problemas menores: } \\
\text { distrair os guardas e encontrar o te- } \\
\text { souro. }\end{array}$ \\
\hline Competência & 01E01CC01 & $\begin{array}{l}\text { Particionamento } \\
\text { Regular }\end{array}$ & $\begin{array}{l}\text { Guiar a divisão de um problema para } \\
\text { que suas partes tenham todas uma } \\
\text { mesma estrutura, podendo portanto } \\
\text { reutilizar soluções. }\end{array}$ & $\begin{array}{l}\text { O uso de um argumento na função } \\
\text { hero.move () permite que o herói } \\
\text { se movimento } \mathrm{N} \text { vezes com um único } \\
\text { comando. (...) alguns jogadores reco- } \\
\text { nheceram (...) e passaram a utilizá-lo. }\end{array}$ \\
\hline $\begin{array}{l}\text { Método de } \\
\text { Ensino }\end{array}$ & 01E01UD01 & $\begin{array}{l}\text { Oficina Code- } \\
\text { Combat }\end{array}$ & \multicolumn{2}{|c|}{$\begin{array}{l}\text { Constituímos nosso estudo de caso a partir de uma Oficina de Introdução à } \\
\text { Ciência da Computação mediada pelo serious game CodeCombat (...) }\end{array}$} \\
\hline Recurso & 01E01TD01 & CodeCombat & \multicolumn{2}{|c|}{$\begin{array}{l}\text { (...) um jogo educativo de aventura em que os jogadores comandam as ações dos } \\
\text { personagens por meio de algoritmos. }\end{array}$} \\
\hline Estudo & 01E01SN01 & $\begin{array}{l}\text { O serious game } \\
\text { CodeCombat } \\
\text { e o professor } \\
\text { como me- } \\
\text { diadores da } \\
\text { aprendizagem } \\
\text { do pensamento } \\
\text { computacional }\end{array}$ & \multicolumn{2}{|c|}{$\begin{array}{l}\text { Este artigo apresenta um recorte de um estudo de caso que analisa uma oficina } \\
\text { mediada pelo serious game CodeCombat, com o objetivo de avaliá-lo como ele- } \\
\text { mento mediador da aprendizagem do pensamento computacional. Utilizam-se } \\
\text { como norteadores teóricos o conceito de mediação, as potencialidades dos seri- } \\
\text { ous games e os princípios do pensamento computacional. Os resultados demons- } \\
\text { tram que o jogo oferece situações que oportunizam o desenvolvimento do pensa- } \\
\text { mento computacional, principalmente da decomposição e generalização. Apesar } \\
\text { disso, a mediação do professor ainda é muito importante para que o aluno inter- } \\
\text { nalize as ações realizadas dentro do jogo. }\end{array}$} \\
\hline
\end{tabular}

A Tabela 3 traz um exemplo de catalogação utilizando o PC-TES sobre um artigo que relata a experiência da utilização do serious game CodeCombat para desenvolver o PC [Severgnini and Soares 2019]. A última coluna da tabela traz trechos de texto retirados diretamente do artigo, quando uma célula mescla as duas últimas colunas, significa que a definição usada na catalogação é diretamente o trecho retirado do artigo. Para poupar 
espaço, as informações que podem ser inferidas pelos códigos dos elementos não são explicitadas na tabela. Por exemplo, a Oficina CodeCombat (01E01UD01) tem como alvo a divisão (01E01), como perspectiva o aluno como Usuário e como categoria atividades Digitais. Destaca-se que no caso do particionamento regular, ele poderia ser considerado uma competência de mais de uma habilidade: decomposição e generalização.

\section{Conclusão}

O sistema PC-TES apresentado neste artigo é uma estrutura de dados com fins de padronização para permitir a conexão de dois ambientes que poderiam desfrutar dos trabalhos realizados pelo outro: o acadêmico e o informal. O PC-TES propõe descrever habilidades do PC por meio de tópicos, expressões e sensibilidades, enaltecendo a separação de manifestações dessas habilidades por sua natureza: que pode ser teórica, de conteúdos aprendidos; ativa, de atitudes tomadas; ou passiva, de sentidos aguçados. É resgatado o conceito de habilidade como um atributo relacionado à performance geral de um indivíduo em relação a um conjunto de tarefas, as quais são apontadas por competências. Portanto, o sistema parece adequado para o objetivo de descrever de maneira padronizada o que é trabalhado no contexto do PC. Assim é aberto o caminho para os anseios do projeto PACT, de realizar uma catalogação na área do PC alimentada tanto por fontes científicas quanto informais. Considera-se promissora a catalogação, visto que de uma rápida análise ilustrativa já é possível extrair informações e inferir habilidades e competências do PC.

É previsto que o objetivo final do PACT possui riscos importantes que devem ser analisados ao longo de sua implementação. O primeiro risco se refere a garantir que, apesar de aberto para entradas informais, não haverá conteúdo errôneo e desinformativo. Para isto, prevê-se que a interface que possibilitará os usuários a submeterem seus relatos e recursos deverá conduzí-lo através de formulários. Além disto, cada conteúdo submetido deve ser revisado pela comunidade e/ou administradores da plataforma e receber selos de verificação, explicitando a confiabilidade do material. O segundo risco se refere à "morte" do repositório, i.e. não ser populado com conteúdo devido à falta de usuários. Um esforço previsto para combater tal risco é fornecer uma população inicial, para que o público ache a plataforma útil e seja incentivado a colaborar. Portanto, prevê-se o desenvolvimento de revisões sistemáticas da literatura, catalogando o que é reportado na comunidade científica em relação ao PC, bem como a submissão de atividades e ferramentas do grupo ExpPC ${ }^{1}$, garantindo uma comunidade para a movimentação inicial da plataforma.

\section{Referências}

Alencar, L. et al. (2020). Criação de um jogo para desenvolver o pensamento computacional percorrendo caminhos eulerianos. In WEI, pages 111-115. SBC.

Bonney, R. et al. (2009). Citizen science: A developing tool for expanding science knowledge and scientific literacy. BioScience, 59(11):977-984.

Brasil (2017). Base nacional comum curricular. Ministério da Educação, 2017. Disponível em: http://basenacionalcomum.mec.gov.br/images/BNCC_EI_EF_ 110518_versaofinal_site.pdf. Acesso em 05 jul. 2019.

Carroll, J. B. et al. (1993). Human cognitive abilities: A survey of factor-analytic studies. Cambridge University Press.

\footnotetext{
${ }^{1}$ https://wp.ufpel.edu.br/pensamentocomputacional/pt/
} 
CIEB (2018). Análise e contribuições para a proposta da bncc-em com foco em tecnologia e computação. Centro de Inovação para a Educação Brasileira, 2018. Disponível em: https://cieb.net.br/wp-content/uploads/2019/06/CIEB-Notas-TAcesso em 05 jul. 2019.

Denning, P. J. and Tedre, M. (2019). Computational Thinking. Mit Press.

Dreyfus, S. E. and Dreyfus, H. L. (1980). A five-stage model of the mental activities involved in directed skill acquisition. Technical report, Univ Berkeley Operations Research Center.

Haines, S. et al. (2019). The effects of computational thinking professional development on stem teachers' perceptions and pedagogical practices. ATHENS JOURNAL OF SCIENCES, 6:97-122.

Kalelioglu, F., Gulbahar, Y., and Kukul, V. (2016). A framework for computational thinking based on a systematic research review. Baltic J. Modern Computing.

Lopes, A. F. et al. (2020). O ensino de pensamento computacional por meio de jogos desplugados e olimpíadas científicas: um relato de experiência nos anos finais do ensino fundamental. In WEI, pages 96-100. SBC.

Lu, J. J. and Fletcher, G. H. (2009). Thinking about computational thinking. In ACM SIGCSE Bulletin, volume 41, pages 260-264. ACM.

MacDonald, E. et al. (2015). Aurorasaurus: A citizen science platform for viewing and reporting the aurora. Space Weather, 13(9):548-559.

Mota, L. P. and Neves, I. (2020). Robótica como ferramenta para o desenvolvimento do pensamento computacional e introdução a lógica de programação. In WEI, pages 141-145. SBC.

Raabe, A. L. A., Brackmann, C. P., and Campos, F. R. (2018). Currículo de tecnologia e computação. CIEB, 2018. Disponível em: https://curriculo.cieb.net.br/assets/docs/Curriculo_de_Referencia_em_Tecnologia_e Computacao.pdf. Acesso em 05 jul. 2019.

Román-González, M., Moreno-León, J., and Robles, G. (2019). Combining assessment tools for a comprehensive evaluation of computational thinking interventions. In Computational Thinking Education, pages 79-98. Springer.

SBC (2018). Diretrizes para o ensino de computação na educação básica. Sociedade Brasileira de Computação, 2018. Disponível em: http://www.sbc.org.br/documentos-dasbc/send/131-curriculos-de-referencia/1177-diretrizes-para-ensino-de-computacaona-educacao-basica. Acesso em 05 jul. 2019.

Severgnini, L. and Soares, E. (2019). O serious game codecombat e o professor como mediadores da aprendizagem do pensamento computacional. In SBIE, page 684.

Silva Junior, B. A. d. (2020). GGasCT: bringing formal methods to the computational thinking. Master's thesis, Universidade Federal de Pelotas.

Vicari, R. M., Moreira, A. F., and Menezes, P. F. B. (2018). Pensamento computacional: revisão bibliográfica. UFRGS, 2018. Disponível em: https://www.lume.ufrgs.br/ bitstream/handle/10183/197566/001097710.pdf?. Acesso em 05 jul. 2019.

Wing, J. M. (2006). Computational thinking. Communications of the ACM, 49(3):33-35. 